\title{
Sumudu Transform Method for Analytical Solutions of Fractional Type Ordinary Differential Equations
}

\author{
Seyma Tuluce Demiray, ${ }^{1}$ Hasan Bulut, ${ }^{1}$ and Fethi Bin Muhammad Belgacem ${ }^{2}$ \\ ${ }^{1}$ Department of Mathematics, Firat University, 23119 Elazig, Turkey \\ ${ }^{2}$ Department of Mathematics, Faculty of Basic Education, PAAET, 92400 Al-Ardiya, Kuwait
}

Correspondence should be addressed to Seyma Tuluce Demiray; seymatuluce@gmail.com

Received 26 June 2014; Revised 5 August 2014; Accepted 5 August 2014

Academic Editor: Abdon Atangana

Copyright (C) 2015 Seyma Tuluce Demiray et al. This is an open access article distributed under the Creative Commons Attribution License, which permits unrestricted use, distribution, and reproduction in any medium, provided the original work is properly cited.

\begin{abstract}
We make use of the so-called Sumudu transform method (STM), a type of ordinary differential equations with both integer and noninteger order derivative. Firstly, we give the properties of STM, and then we directly apply it to fractional type ordinary differential equations, both homogeneous and inhomogeneous ones. We obtain exact solutions of fractional type ordinary differential equations, both homogeneous and inhomogeneous, by using STM. We present some numerical simulations of the obtained solutions and exhibit two-dimensional graphics by means of Mathematica tools. The method used here is highly efficient, powerful, and confidential tool in terms of finding exact solutions.
\end{abstract}

\section{Introduction}

Computation and analysis of solutions for nonlinear fractional differential equations now span a half-century or more and play a crucial role in several theoretical and applied sciences such as, but certainly not limited to, theoretical biology and ecology, solid state physics, viscoelasticity, fiber optics, signal processing and electric control theory, stochastic based finance, and hemo-, hydro-, and thermodynamics [1-7]. In particular, modified Kudryashov method [8], generalized Kudryashov method (GKM) [9], adomian decomposition method (ADM) [10], and homotopy decomposition method (HDM) [11] have all been used to seek solutions to fractional differential equations.

The Sumudu transform method (STM) was initiated in 1993, by Watugala who used it to solve engineering control problems $[12,13]$. Subsequently, he expanded the Sumudu transform to two variables [14]. The first application of the STM to partial differential equations and the establishment of the inverse formula were done by Weerakoon $[15,16]$. Sumudu transform based solutions to convolution type integral equations and discrete dynamic systems were later obtained by Asiru [17-19]. Following in this trend, Belgacem et al. established applications of the STM [20, 21]. Most of the STM applications in the first decade of the 21st century concentrated on integer type differential equations, and although Belgacem's practical interests for applying to fractional differential equations started as early as 2008 in various local presentations and conference talks, concrete results in this direction started appearing only in the second decade, with various teams of research [22], including the Bulut et al. team [23]. In fact, the Bulut et al. teams of research have been most active in the integer type differential equations area using the STM $[24,25]$. Also, Atangana and Kilicman have tackled Sumudu transform method for nonlinear fractional heat-like equations [26].

In this work, our aim is to exhibit exact solutions of some homogeneous and nonhomogeneous fractional ordinary differential equations by using the STM. For paper layout, we first recall basic features of fractional calculus, in Section 2. In Section 3, we remind the reader of the properties of the STM. In Section 4, we apply the STM to obtain new exact solutions of both homogeneous and inhomogeneous fractional ordinary differential equations. 


\section{Basic Features of Fractional Calculus}

In this section, we primarily introduce main features of fractional calculus following notations [1-7], making consensus among them. Fractional derivatives and integrals are most commonly introduced and used in the sense of AbelRiemann (A-R), described by

$$
D^{\alpha} f(t)= \begin{cases}\frac{1}{\Gamma[m-\alpha]} \frac{d}{d t^{m}} \int_{0}^{t} \frac{f(\tau)}{(t-\tau)^{\alpha-m+1}} d \tau, \\ \frac{m-1<\alpha<m,}{\frac{d^{m}}{d t^{m}} f(t),} & \alpha=m,\end{cases}
$$

where $m \in \mathbb{Z}^{+}, \alpha \in R^{+}$. $D^{\alpha}$ is derivative operator and

$$
D^{-\alpha} f(t)=\frac{1}{\Gamma[\alpha]} \int_{0}^{t}(t-\tau)^{\alpha-1} f(\tau) d \tau, \quad 0<\alpha \leq 1 .
$$

Also, according to A-R, an integral of fractional order is described by performing the integration operator $J^{\alpha}$ in the following format:

$$
J^{\alpha} f(t)=\frac{1}{\Gamma[\alpha]} \int_{0}^{t}(t-\tau)^{\alpha-1} f(\tau) d \tau, \quad t>0, \alpha>0 .
$$

Following Podlubny [3] set-up, we have

$$
\begin{aligned}
J^{\alpha} t^{n} & =\frac{\Gamma[1+n]}{\Gamma[1+n+\alpha]} t^{n+\alpha}, \\
D^{\alpha} t^{n} & =\frac{\Gamma[1+n]}{\Gamma[1+n-\alpha]} t^{n-\alpha} .
\end{aligned}
$$

Another important and basic definition was first established by Caputo [1], given by

$$
{ }^{C} D^{\alpha} f(t)= \begin{cases}\frac{1}{\Gamma[m-\alpha]} \int_{0}^{t} \frac{f^{(m)}(\tau)}{(t-\tau)^{\alpha-m+1}} d \tau, & m-1<\alpha<m, \\ \frac{d^{m}}{d t^{m}} f(t), & \alpha=m .\end{cases}
$$

A basic property of the Caputo fractional derivative is that (see for instance [22])

$$
J^{\alpha}\left[{ }^{C} D^{\alpha} f(t)\right]=f(t)-\sum_{k=0}^{\infty} f^{(k)}\left(0^{+}\right) \frac{t^{k}}{k !} .
$$

\section{Basic Features of the Sumudu Transform Method (STM)}

The Sumudu transform is obtained over the set of functions $[20]$ as

$$
\begin{gathered}
A=\left\{f(t)\left|\exists M, \tau_{1}, \tau_{2}>0,\right| f(t) \mid<M e^{|t| / \tau_{i}},\right. \\
\text { if } \left.t \in(-1)^{j} \times[0, \infty)\right\}
\end{gathered}
$$

by

$$
F(u)=S[f(t)]=\int_{0}^{\infty} f(u t) e^{-t} d t, \quad u \in\left(-\tau_{1}, \tau_{2}\right) .
$$

Theorem 1. If $F(u)$ is Sumudu transform of $f(t)$, then the Sumudu transform of the derivatives with integer order is as follows [20-23]:

$$
S\left[\frac{d^{n} f(t)}{d t^{n}}\right]=u^{-n}\left[F(u)-\left.\sum_{k=0}^{n-1} u^{k} \frac{d^{n} f(t)}{d t^{n}}\right|_{t=0}\right] .
$$

Proof. The Sumudu transform of the first derivative of $f(t)$, $f^{\prime}(t)=d f(t) / d t$, is given by

$$
\begin{aligned}
S\left[\frac{d f(t)}{d t}\right]= & \int_{0}^{\infty} \frac{d f(u t)}{d t} e^{-t} d t=\lim _{p \rightarrow \infty} \int_{0}^{p} \frac{d f(u t)}{d t} e^{-t} d t \\
= & \lim _{p \rightarrow \infty}\left[\left.\frac{1}{u} e^{-t / u} f(t)\right|_{0} ^{p}+\frac{1}{u^{2}} \int_{0}^{p} e^{-t / u} f(t) d t\right] \\
= & \lim _{p \rightarrow \infty}\left[\left.\frac{1}{u} e^{-t / u} f(t)\right|_{0} ^{p}\right. \\
& \left.\quad+\frac{1}{u}\left(\frac{1}{u} \int_{0}^{p} e^{-t / u} f(t) d t\right)\right] \\
= & \lim _{p \rightarrow \infty}\left[-\frac{1}{u} f(0)+\frac{1}{u}\left(\frac{1}{u} \int_{0}^{p} e^{-t / u} f(t) d t\right)\right] \\
= & -\frac{1}{u} f(0)+\frac{1}{u} F(u) .
\end{aligned}
$$

Proceeding in the same manner, we get the Sumudu transform of the second order derivative as

$$
S\left[\frac{d^{2} f(t)}{d t^{2}}\right]=\frac{1}{u^{2}}\left[F(u)-f(0)-\left.u \frac{d f(t)}{d t}\right|_{t=0}\right] .
$$

To finish the proof of Theorem 1, we proceed by induction in the same way only to reach the general formula for the Sumudu transform of any integer $n$-order derivative [23]; namely,

$$
S\left[\frac{d^{n} f(t)}{d t^{n}}\right]=u^{-n}\left[F(u)-\left.\sum_{k=0}^{n-1} u^{k} \frac{d^{n} f(t)}{d t^{n}}\right|_{t=0}\right] .
$$

Theorem 2. If $F(u)$ is the Sumudu transform of $f(t)$, then the Sumudu transform of the Riemann-Liouville fractional derivative is given by (see [22])

$$
\begin{array}{r}
S\left[D^{\alpha} f(t)\right]=u^{-\alpha}\left[F(u)-\sum_{k=1}^{n} u^{\alpha-k}\left[D^{\alpha-k}(f(t))\right]_{t=0}\right], \\
-1<n-1 \leq \alpha<n .
\end{array}
$$

At the moment, we harness the properties developments of the STM and utilize them for finding exact solutions of fractional ordinary differential equations.

We consider the general linear fractional ordinary differential equation (FODE) as follows:

$$
\frac{\partial^{\alpha} y(t)}{\partial t^{\alpha}}=\frac{\partial^{2} y(t)}{\partial t^{2}}+\frac{\partial y(t)}{\partial t}+y(t)+c,
$$


subject to the initial condition

$$
y(0)=f(0) .
$$

When we get Sumudu transform of (14) taking into consideration (12) and (13), we obtain Sumudu transform of (14) as follows:

$$
\begin{aligned}
& S\left[\frac{\partial^{\alpha} y(t)}{\partial t^{\alpha}}\right]=S\left[\frac{\partial^{2} y(t)}{\partial t^{2}}\right]+S\left[\frac{\partial y(t)}{\partial t}\right]+S[y(t)]+S[c], \\
& u^{-\alpha}\left[F(u)-\sum_{k=1}^{n} u^{\alpha-k}\left[D^{\alpha-k}(f(t))\right]_{t=0}\right] \\
& =\frac{1}{u^{2}}\left[F(u)-f(0)-\left.u \frac{\partial f(t)}{\partial t}\right|_{t=0}\right] \\
& +\frac{1}{u}[F(u)-f(0)]+F(u)+c, \\
& F(u)-\sum_{k=1}^{n} u^{\alpha-k}\left[D^{\alpha-k}(f(t))\right]_{t=0} \\
& =u^{\alpha-2}\left[F(u)-f(0)-\left.u \frac{\partial f(t)}{\partial t}\right|_{t=0}\right] \\
& +u^{\alpha-1}[F(u)-f(0)]+u^{\alpha} F(u)+c u^{\alpha}, \\
& F(u)=u^{\alpha-2} F(u)-u^{\alpha-2} f(0)+\sum_{k=1}^{n} u^{\alpha-k}\left[D^{\alpha-k}(f(t))\right]_{t=0} \\
& -\left.u^{\alpha-1} \frac{\partial f(t)}{\partial t}\right|_{t=0}+u^{\alpha-1} F(u)-u^{\alpha-1} f(0) \\
& +u^{\alpha} F(u)+c u^{\alpha}, \\
& F(u)-u^{\alpha-2} F(u)-u^{\alpha-1} F(u)-u^{\alpha} F(u) \\
& =-u^{\alpha-2} f(0)+\sum_{k=1}^{n} u^{\alpha-k}\left[D^{\alpha-k}(f(t))\right]_{t=0} \\
& -\left.u^{\alpha-1} \frac{\partial f(t)}{\partial t}\right|_{t=0}-u^{\alpha-1} f(0)+c u^{\alpha} \\
& F(u)=\frac{1}{1-u^{\alpha-2}-u^{\alpha-1}-u^{\alpha}} \\
& \times\left[g(u)-u^{\alpha-1} f(0)-u^{\alpha-2} f(0)+c u^{\alpha}\right] \text {, }
\end{aligned}
$$

where $g(u)$ is described by $\sum_{k=1}^{n} u^{\alpha-k}\left[D^{\alpha-k}(f(t))\right]_{t=0}-$ $\left.u^{\alpha-1}(\partial f(t) / \partial t)\right|_{t=0}$.
When we get inverse Sumudu transform of (16) by using inverse transform table in $[21,22]$, we obtain solution of (14) by using STM in the following manner:

$$
\begin{aligned}
y(t)=S^{-1}[ & \frac{1}{1-u^{\alpha-2}-u^{\alpha-1}-u^{\alpha}} \\
& \left.\times\left[g(u)-u^{\alpha-1} f(0)-u^{\alpha-2} f(0)+c u^{\alpha}\right]\right] .
\end{aligned}
$$

\section{STM Implementations to Homogeneous and Inhomogeneous FODEs}

In this Section, we implement the STM to homogeneous and inhomogeneous fractional ordinary differential equations (HFODEs and IHFODEs) in the following three examples.

Example 3. Initially, we consider the inhomogeneous fractional ordinary differential equation [4] as

$$
\begin{aligned}
D^{2} y(t)+D^{3 / 2} y(t)+y(t)=t+1, \quad\left[{ }_{0} D_{t}^{-1 / 2} y(t)\right]_{t=0} & =0, \\
t & >0,
\end{aligned}
$$

subject to the initial conditions

$$
y(0)=y^{\prime}(0)=1 .
$$

In order to find exact solution of (18), we take the Sumudu transform of both sides of (18) as follows:

$$
\begin{gathered}
S\left[D^{2} y(t)\right]+S\left[D^{3 / 2} y(t)\right]+S[y(t)]=S[t]+S[1] \\
\frac{1}{u^{2}}\left[F(u)-f(0)-\left.u \frac{d f(t)}{d t}\right|_{t=0}\right]+\frac{F(u)}{u^{3 / 2}}-\left.\frac{D^{1 / 2}[f(t)]}{u}\right|_{t=0} \\
\quad+F(u)=u+1, \\
\frac{1}{u^{2}}[F(u)-1-u]+\frac{F(u)}{u^{3 / 2}}-\frac{1}{u}\left[\frac{F(u)}{u^{1 / 2}}-\left.\frac{D^{-1 / 2}[f(t)]}{u}\right|_{t=0}\right] \\
\quad+F(u)=u+1, \\
\frac{1}{u^{2}}[F(u)-1-u]+\left.\frac{D^{-1 / 2}[f(t)]}{u}\right|_{t=0}+F(u)=u+1 \\
\left(1+\frac{1}{u^{2}}\right) F(u)=1+u+\frac{1}{u}+\frac{1}{u^{2}} \\
\Longrightarrow\left(1+u^{2}\right) F(u)=1+u+u^{2}+u^{3} \\
F(u)=\frac{1+u+u^{2}+u^{3}}{\left(1+u^{2}\right)}=1+u .
\end{gathered}
$$

Upon Sumudu inverting (20) which is linear (also see inverse transform table in [21]), we obtain exact solution as

$$
y(t)=1+t
$$


Remark 4. The exact solution of (18) found here by using Sumudu transform method agrees with the solution in [4] for corresponding values of tuned parameters.

Example 5. Secondly, we consider homogeneous fractional ordinary differential equation as follows [6]:

$$
\begin{array}{r}
\frac{d y(t)}{d t}+\frac{d^{1 / 2} y(t)}{d t^{1 / 2}}-2 y(t)=0, \quad \frac{d^{1 / 2} y\left(0^{+}\right)}{d t^{1 / 2}}=c, \\
t>0
\end{array}
$$

with initial condition

$$
y\left(0^{+}\right)=0 .
$$

We first apply the Sumudu transform of both sides of (22) to get

$$
S\left(\frac{d y(t)}{d t}\right)+S\left(\frac{d^{1 / 2} y(t)}{d t^{1 / 2}}\right)-2 S(y(t))=0
$$

which yields

$$
\begin{gathered}
\frac{1}{u}[F(u)-f(0)]+\frac{F(u)}{u^{1 / 2}}-\left.\frac{D^{-1 / 2}[f(t)]}{u}\right|_{t=0}-2 F(u)=0, \\
\frac{F(u)}{u}+\frac{F(u)}{u^{1 / 2}}-\frac{c}{u}-2 F(u)=0 \\
\left(1+u^{1 / 2}-2 u\right) F(u)=c \\
\Longrightarrow F(u)=\frac{c}{1+u^{1 / 2}-2 u} \\
=\frac{2 c}{3(1+2 \sqrt{u})}+\frac{c}{3(1-\sqrt{u})} \\
F(u)=\frac{2 c}{3}\left(\frac{1-2 \sqrt{u}}{1-4 u}\right)+\frac{c}{3}\left(\frac{1+\sqrt{u}}{1-u}\right), \\
F(u)=\frac{2 c}{3}\left(\frac{1}{1-4 u}-\frac{2 \sqrt{u}}{1-4 u}\right)+\frac{c}{3}\left(\frac{1}{1-u}-\frac{\sqrt{u}}{1-u}\right) .
\end{gathered}
$$

When we take inverse Sumudu transform of (25) by using inverse transform table in [21], we obtain exact solution of (22) by using STM as follows:

$$
y(t)=\frac{2 c}{3}\left[e^{4 t}-e^{4 t} \operatorname{erf}(2 \sqrt{t})\right]+\frac{c}{3}\left[e^{t}+e^{t} \operatorname{erf}(\sqrt{t})\right] .
$$

Remark 6. To our knowledge, the solution of (22), obtained by using the STM, is new and does not figure in the published literature.

Example 7. Thirdly, we investigate the inhomogeneous fractional ordinary differential equation [6] as

$$
\begin{aligned}
{ }_{0} D_{t}^{1 / 2} y(t)-y(t)=-1, \quad\left[{ }_{0} D_{t}^{-1 / 2} y(t)\right]_{t=0} & =0, \\
t & >0 .
\end{aligned}
$$

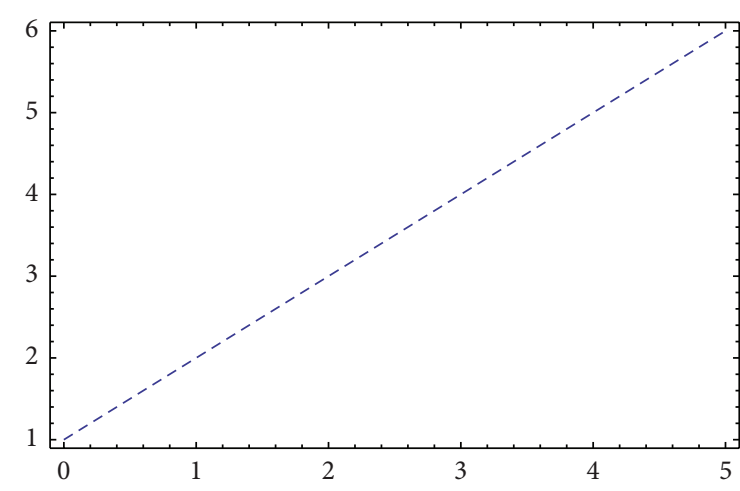

FIGURE 1: Two-dimensional graphic for exact solution of (18), when $0<t<5$, via the STM.

In order to obtain the exact solution of (27) by using the STM, we apply the Sumudu transform to both sides of (27) as follows:

$$
\begin{gathered}
S\left[D^{1 / 2} y(t)\right]-S[y(t)]+S[1]=0, \\
S\left[D^{1 / 2} y(t)\right]-F(u)=-S[1], \\
\frac{F(u)}{u^{1 / 2}}-\left.\frac{D^{-1 / 2}[f(t)]}{u}\right|_{t=0}-F(u)=-S[1], \\
\frac{F(u)}{u^{1 / 2}}-F(u)=-1 \\
\Longrightarrow\left(\frac{1}{u^{1 / 2}}-1\right) F(u)=-1 \\
\Longrightarrow\left(1-u^{1 / 2}\right) F(u)=-u^{1 / 2}, \\
F(u)=\frac{\sqrt{u}}{\sqrt{u}-1}=\frac{\sqrt{u}(\sqrt{u}+1)}{u-1}=\frac{u+\sqrt{u}}{u-1} \\
=\frac{u-1+1+\sqrt{u}}{u-1}=1-\frac{1}{1-u}-\frac{\sqrt{u}}{1-u} .
\end{gathered}
$$

When we get the inverse Sumudu transform of (28) by using the table in [21], we find exact solution of (27) by the STM as follows:

$$
y(t)=1-e^{t}-e^{t} \operatorname{erf} \sqrt{t}
$$

Remark 8. Again, the exact solution of (27) exhibited here, found through implementing the Sumudu transform method, is to our knowledge new and not trackable in previous literature.

We plot solution (21) of (18) in Figure 1, solution (26) of (22) in Figure 2, solution (29) of (27) in Figure 3, which shows the dynamics of solutions with suitable parametric choices.

\section{Conclusions}

In this paper, we considered homogeneous and inhomogeneous fractional ordinary differential equations (HFODEs and IHFODEs) and treated them by applying the Sumudu 


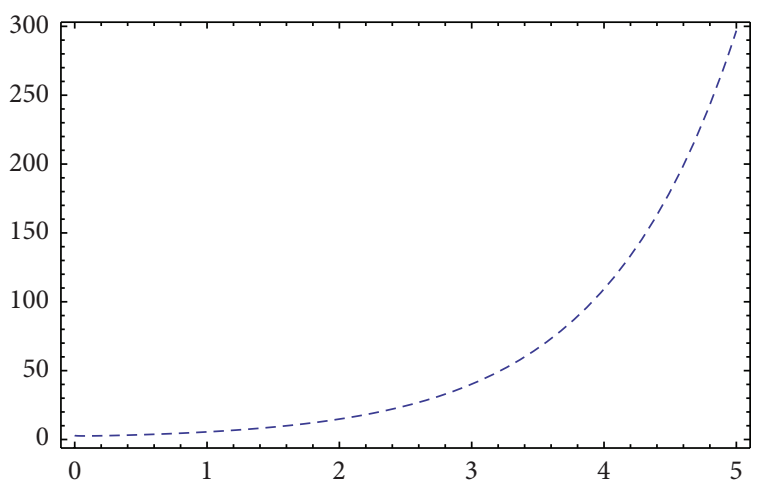

Figure 2: Two-dimensional graphic for exact solution of (22), for $0<t<5$ and $c=3$, via the STM.

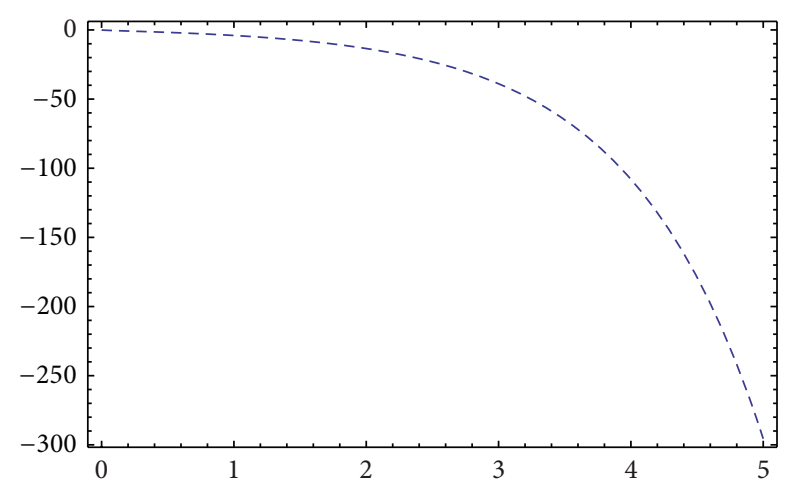

Figure 3: Two-dimensional graphic for exact solution of (27), for $0<t<5$, via the STM.

transform method and obtained their exact solutions. To date and to our knowledge, the exact solutions obtained in the last two examples are new. We also exhibited two-dimensional graphics for the obtained solutions by means of programming language Mathematica. Accordingly, we can conclude that not only does the Sumudu transform method play an important role in treating homogeneous and inhomogeneous fractional ordinary differential equations, but also it is highly effective with regard to yielding exact solutions. We expect the STM to be equally successful when treating even more complex applications than presented involving FODEs.

\section{Conflict of Interests}

The authors declare that there is no conflict of interests regarding the publication of this paper.

\section{References}

[1] M. Caputo, "Linear model of dissipation whose Q is almost frequency independent-II," Geophysical Journal of the Royal Astronomical Society, vol. 13, no. 5, pp. 529-539, 1967.

[2] K. S. Miller and B. Ross, An Introduction to the Fractional Calculus and Fractional Differantial Equations, John Wiley \& Sons, New York, NY, USA, 1993.
[3] I. Podlubny, Fractional Differantial Equations, Academic Press, San Diego, Calif, USA, 1999.

[4] K. Diethelm and Y. Luchko, "Numerical solution of linear multi-term initial value problems of fractional order," Journal of Computational Analysis and Applications, vol. 6, no. 3, pp. 243263, 2004.

[5] A. A. Kilbas, H. M. Srivastava, and J. J. Trujillo, Theory and Applications of Fractional Differential Equations, Elsevier Science, New York, NY, USA, 2006.

[6] R. L. Magin, Fractional Calculus in Bioengineering, Begell House, Redding, Conn, USA, 2006.

[7] R. C. Mittal and R. Nigam, "Solution of fractional integrodifferential equations by Adomian decomposition method," The International Journal of Applied Mathematics and Mechanics, vol. 4, no. 2, pp. 87-94, 2008.

[8] Y. A. Tandogan, Y. Pandir, and Y. Gurefe, "Solutions of the nonlinear differential equations by use of modified Kudryashov method," Turkish Journal of Mathematics and Computer Science, vol. 2013, Article ID 20130021, 7 pages, 2013.

[9] S. Tuluce Demiray, Y. Pandir, and H. Bulut, "Generalized kudryashov method for time-fractional differential equations," Abstract and Applied Analysis, vol. 2014, Article ID 901540, 13 pages, 2014.

[10] G. Adomian, "A review of the decomposition method and some recent results for nonlinear equations," Mathematical and Computer Modelling, vol. 13, no. 7, pp. 17-43, 1990.

[11] A. Atangana, S. Tuluce Demiray, and H. Bulut, "Modelling the nonlinear wave motion within the scope of the fractional calculus," Abstract and Applied Analysis, vol. 2014, Article ID 481657, 7 pages, 2014.

[12] G. K. Watugala, "Sumudu transform: a new integral transform to solve differential equations and control engineering problems," International Journal of Mathematical Education in Science and Technology, vol. 24, no. 1, pp. 35-43, 1993.

[13] G. K. Watugala, "Sumudu transform-a new integral transform to solve differential equations and control engineering problems," Mathematical Engineering in Industry, vol. 6, no. 4, pp. 319-329, 1998.

[14] G. K. Watugala, "The Sumudu transform for functions of two variables," Mathematical Engineering in Industry, vol. 8, no. 4, pp. 293-302, 2002.

[15] S. Weerakoon, "Application of Sumudu transform to partial differential equations," International Journal of Mathematical Education in Science and Technology, vol. 25, no. 2, pp. 277-283, 1994.

[16] S. Weerakoon, "Complex inversion formula for Sumudu transform," International Journal of Mathematical Education in Science and Technology, vol. 29, no. 4, pp. 618-621, 1998.

[17] M. A. Asiru, "Sumudu transform and the solution of integral equations of convolution type," International Journal of Mathematical Education in Science and Technology, vol. 32, no. 6, pp. 906-910, 2001.

[18] M. A. Asiru, "Further properties of the Sumudu transform and its applications," International Journal of Mathematical Education in Science and Technology, vol. 33, no. 3, pp. 441-449, 2002.

[19] M. A. Asiru, "Application of the sumudu transform to discrete dynamical systems," International Journal of Mathematical Education in Science and Technology, vol. 34, no. 6, pp. 944-949, 2003. 
[20] F. B. M. Belgacem, A. A. Karaballi, and S. L. Kalla, "Analytical investigations of the Sumudu transform and applications to integral production equations," Mathematical Problems in Engineering, vol. 2003, no. 3, pp. 103-118, 2003.

[21] F. B. M. Belgacem and A. A. Karaballi, "Sumudu transform fundamental properties investigations and applications," Journal of Applied Mathematics and Stochastic Analysis, vol. 2006, Article ID 91083, 23 pages, 2006.

[22] Q. D. Katatbeh and F. B. M. Belgacem, "Applications of the Sumudu transform to fractional differential equations," Nonlinear Studies, vol. 18, no. 1, pp. 99-112, 2011.

[23] H. Bulut, H. M. Baskonus, and F. B. M. Belgacem, "The analytical solution of some fractional ordinary differential equations by the Sumudu transform method," Abstract and Applied Analysis, vol. 2013, Article ID 203875, 6 pages, 2013.

[24] H. Bulut, H. M. Baskonus, and S. Tuluce, "The solutions of partial differential equations with variable coefficient by Sumudu transform method," in Proceedings of the 9th International Conference on Mathematical Problems in Engineering, Aerospace and Sciences (ICNPAA '12), vol. 1493 of AIP Proceedings, p. 91, Vienna, Austria, July 2012.

[25] H. Bulut, H. M. Baskonus, and S. Tuluce, "Homotopy perturbation Sumudu transform method for heat equations," Mathematics in Engineering, Science and Aerospace, vol. 4, no. 1, pp. 49-60, 2013.

[26] A. Atangana and A. K1lıçman, "The use of Sumudu transform for solving certain nonlinear fractional heat-like equations," Abstract and Applied Analysis, vol. 2013, Article ID 737481, 12 pages, 2013. 


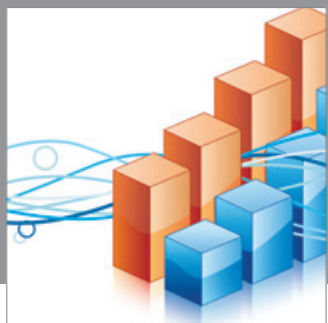

Advances in

Operations Research

mansans

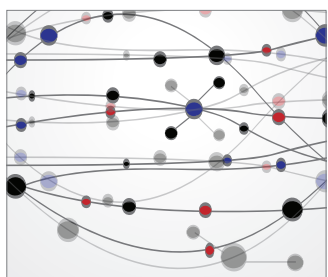

The Scientific World Journal
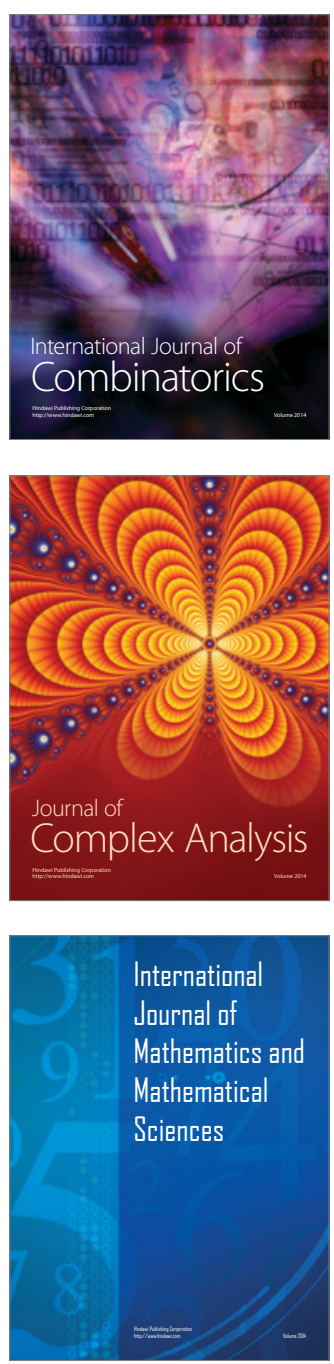
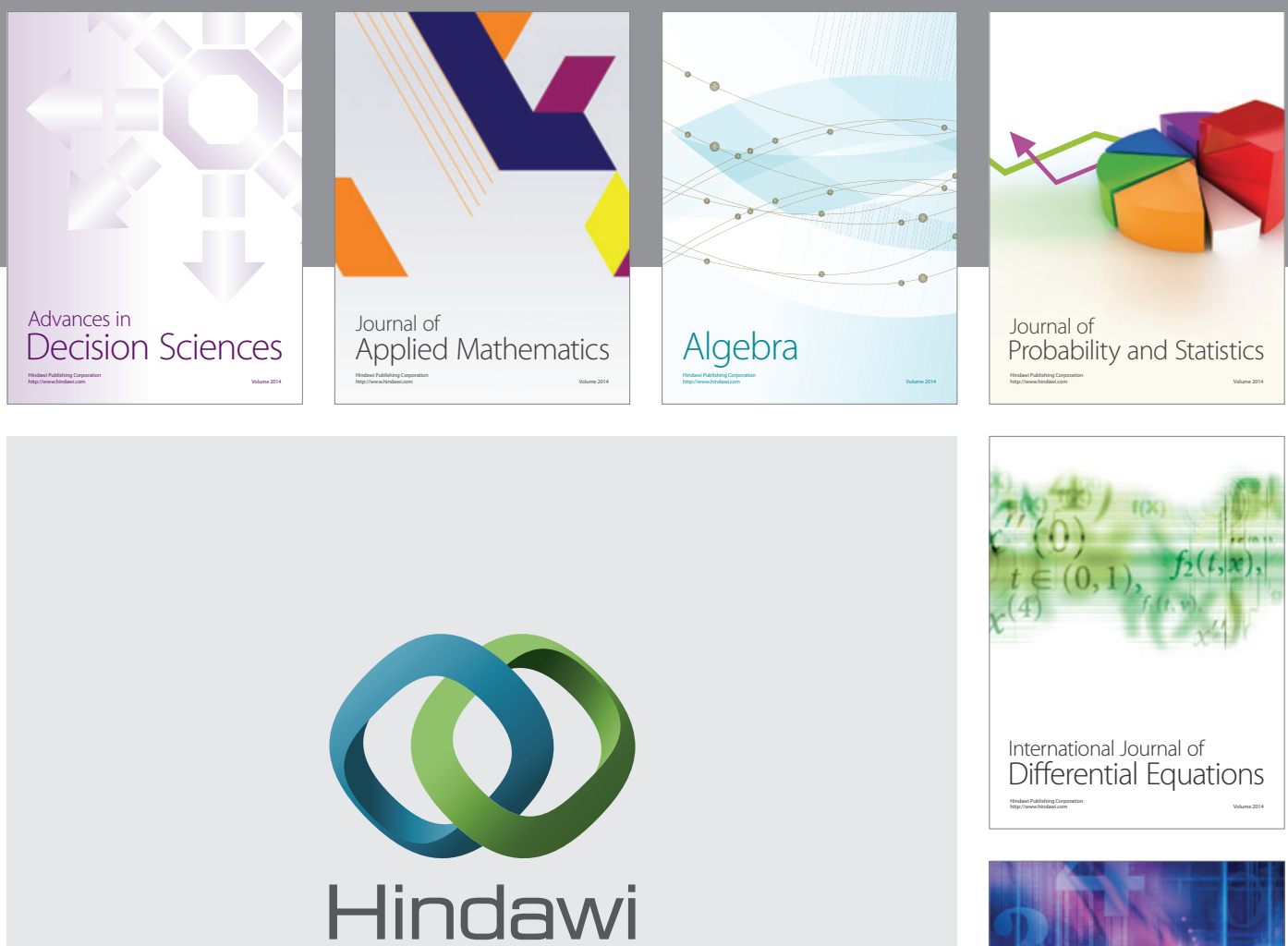

Submit your manuscripts at http://www.hindawi.com
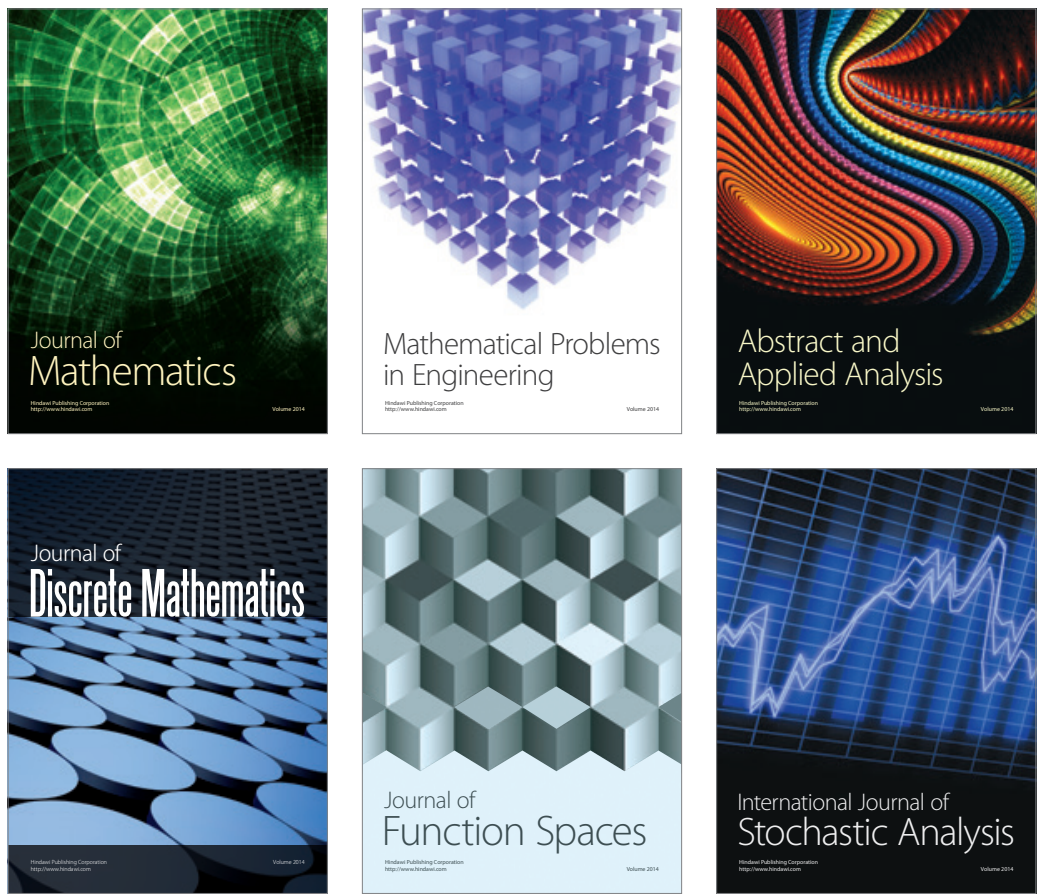

Journal of

Function Spaces

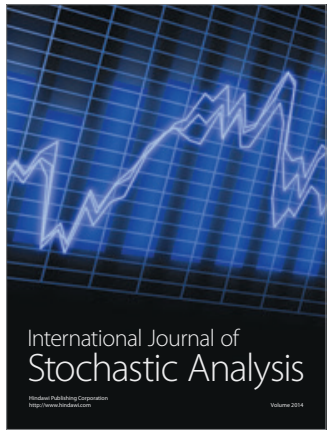

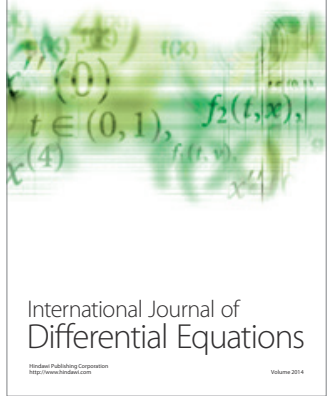
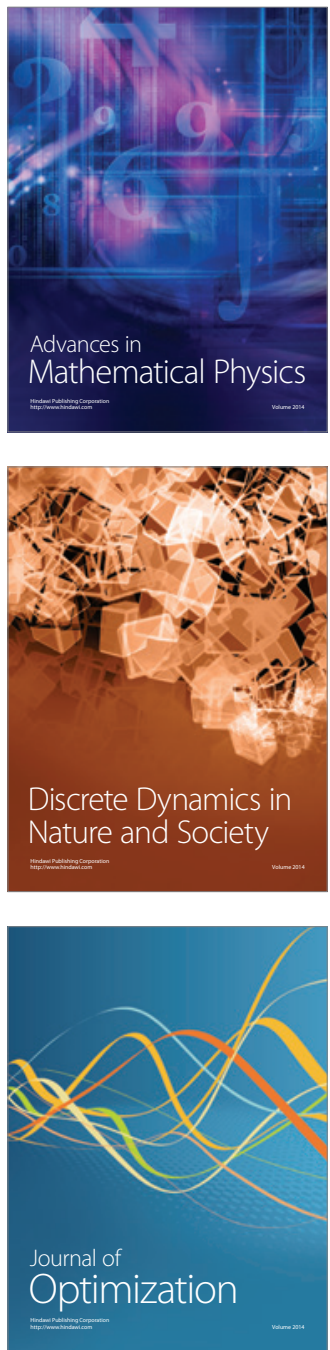